\title{
Investigation of gene-environment interactions in relation to tic severity
}

\author{
Mohamed Abdulkadir ${ }^{1,2} \oplus$. Dongmei $\mathrm{Yu}^{3,4} \cdot$ Lisa Osiecki ${ }^{4} \cdot$ Robert A. King $^{5} \cdot$ Thomas V. Fernandez $^{5}$. \\ Lawrence W. Brown ${ }^{6} \cdot$ Keun-Ah Cheon $^{7}$. Barbara J. Coffey ${ }^{8,9,10}$. Blanca Garcia-Delgar ${ }^{11}$. Donald L. Gilbert ${ }^{12}$. \\ Dorothy E. Grice ${ }^{8}$. Julie Hagstrøm ${ }^{13} \cdot$ Tammy Hedderly $^{14}$. Isobel Heyman ${ }^{15}$. Hyun Ju Hong ${ }^{16}$. Chaim Huyser ${ }^{17}$. \\ Laura Ibanez-Gomez ${ }^{18}$. Young Key Kim ${ }^{19}$. Young-Shin Kim ${ }^{20}$. Yun-Joo Koh ${ }^{21}$. Sodahm Kook ${ }^{22}$. \\ Samuel Kuperman ${ }^{23}$ - Bennett Leventhal ${ }^{20} \cdot$ Marcos Madruga-Garrido $^{24}$ - Athanasios Maras ${ }^{25} \cdot$ Pablo Mir $^{26}$. \\ Astrid Morer ${ }^{27}$. Alexander Münchau ${ }^{28} \cdot$ Kerstin J. Plessen ${ }^{13} \cdot$ Veit Roessner $^{29}$. Eun-Young Shin ${ }^{7}$. Dong-Ho Song ${ }^{30}$. \\ Jungeun Song ${ }^{31}$. Frank Visscher ${ }^{32}$. Samuel H. Zinner ${ }^{33}$. Carol A. Mathews ${ }^{34}$ - Jeremiah M. Scharf ${ }^{3,4}$. \\ Jay A. Tischfield ${ }^{2} \cdot$ Gary A. Heiman $^{2} \cdot$ Andrea Dietrich $^{1} \cdot$ Pieter J. Hoekstra ${ }^{1}$
}

Received: 21 May 2021 / Accepted: 28 July 2021 / Published online: 13 August 2021

(c) The Author(s) 2021

\begin{abstract}
Tourette syndrome (TS) is a neuropsychiatric disorder with involvement of genetic and environmental factors. We investigated genetic loci previously implicated in Tourette syndrome and associated disorders in interaction with pre- and perinatal adversity in relation to tic severity using a case-only $(N=518)$ design. We assessed 98 single-nucleotide polymorphisms (SNPs) selected from (I) top SNPs from genome-wide association studies (GWASs) of TS; (II) top SNPs from GWASs of obsessive-compulsive disorder (OCD), attention-deficit/hyperactivity disorder (ADHD), and autism spectrum disorder (ASD); (III) SNPs previously implicated in candidate-gene studies of TS; (IV) SNPs previously implicated in OCD or ASD; and (V) tagging SNPs in neurotransmitter-related candidate genes. Linear regression models were used to examine the main effects of the SNPs on tic severity, and the interaction effect of these SNPs with a cumulative pre- and perinatal adversity score. Replication was sought for SNPs that met the threshold of significance (after correcting for multiple testing) in a replication sample $(N=678)$. One SNP (rs7123010), previously implicated in a TS meta-analysis, was significantly related to higher tic severity. We found a gene-environment interaction for rs6539267, another top TS GWAS SNP. These findings were not independently replicated. Our study highlights the future potential of TS GWAS top hits in gene-environment studies.
\end{abstract}

Keywords Gene-environment interaction · Pre- and perinatal complications $\cdot$ Tic severity $\cdot$ Tourette syndrome

\section{Introduction}

Tourette syndrome (TS) is a childhood onset neuropsychiatric disorder influenced by both genetic and environmental factors (Robertson et al. 2017). There is clear evidence that implicates both common and rare variants in TS (Qi et al. 2017; Yu et al. 2019); however, specific genetic variants only account for a small proportion of total TS disease

Andrea Dietrich and Pieter J. Hoekstra have contributed equally to this work.

Mohamed Abdulkadir

mohamedabdulkadir@gmail.com

Extended author information available on the last page of the article risk. We investigated the involvement of common SNPs in candidate genes previously implicated in TS and top SNPs from GWAS of TS and comorbid disorders, and found no convincing support for these common variants (Abdulkadir et al. 2018). However, we cannot rule out that these common SNPs might yet confer risk for TS through interaction with environmental factors. Currently, gene-environment (GxE) studies are lacking and only a few small-sampled studies have investigated the genetic etiology of tic severity, suggesting involvement of the dopamine transporter gene (Tarnok et al. 2007) and the dopamine receptor D2 gene (Comings et al. 1991). Unfortunately, no GxE studies have attempted to replicate these initial findings (Qi et al. 2017). Environmental risk factors such as pre- and perinatal risk factors are also implicated in TS (Mathews et al. 2014); two 
studies suggested a role for a cumulative score of adverse pre- and perinatal events in TS (Abdulkadir et al. 2016; Brander et al. 2018).

The aim of the present study was to investigate whether previously implicated SNPs from genome-wide association studies and candidate-gene studies, alone and in interaction with a cumulative pre- and perinatal adversity score, are associated with lifetime tic severity using TS cases recruited by the Tourette International Collaborative Genetics (TIC Genetics) study (Dietrich et al. 2015).

\section{Methods}

\section{Study subjects}

This study included 586 cases $(66.7 \%$ male; mean age 23.6 years, $\mathrm{SD}=16.7$, range 3-79 years) affected with a chronic tic disorder (458 with TS and 128 with chronic motor or vocal tic disorder) from the ongoing TIC Genetics study (Dietrich et al. 2015). As a replication sample, subjects were utilized from the first published TS GWAS (Scharf et al. 2013), including 678 cases ( $77 \%$ male; mean age 18.8 years, $\mathrm{SD}=14$, range $4-78$ years) diagnosed with TS (Scharf et al. 2013).

All adult participants and parents of children provided written informed consent along with written or oral assent of their participating child. The Institutional Review Board of each participating site had approved the study.

\section{Diagnostic assessment}

Lifetime worst-ever tic severity (mean 15.6; $\mathrm{SD}=8.22$, range 0-30) was assessed based on a modified version of the Yale Global Tic Severity Scale (Dietrich et al. 2015). The replication sample included additional items (i.e., number of tics, complexity of tics, and impairment). The mean of both parents' education level was used as a proxy for socioeconomic status (SES).

\section{Cumulative pre- and perinatal adversity score}

A cumulative pre- and perinatal adversity score (mean 3.52; $\mathrm{SD}=3.42$, observed range $0-21$; previously described in (Abdulkadir et al. 2016)) was constructed from addition of 38 possible adverse events as measured by the self-report or parent-on-child report version of the Modified Schedule for Risk and Protective Factors Early in Development questionnaire (Walkup and Leckman 1988). Missing values were categorized as absent (coded as 0 ). The replication sample (Scharf et al. 2013) used the same questionnaire (Walkup and Leckman 1988) in constructing the cumulative perinatal adversity score.

\section{Selection of single-nucleotide polymorphisms}

Genetic variants were selected based on a literature review and described in detail elsewhere (Abdulkadir et al. 2018). Briefly, a total of 196 SNPs were assessed: 12 top SNPs from the prior TS GWAS (Scharf et al. 2013; Paschou et al. 2014); 17 top SNPs from GWAS of obsessive-compulsive disorder (OCD; Stewart et al. 2013), attention-deficit/hyperactivity disorder (ADHD; Mick et al. 2011; Hinney et al. 2011), and autism spectrum disorder (ASD; Wang et al. 2009; Anney et al. 2012); 17 SNPs from candidate genes previously implicated $(P<0.05$; Abdulkadir et al. 2018) in TS; 2 individual candidate SNPs implicated in OCD and one in ASD (Abdulkadir et al. 2018); and 148 tagging SNPs covering seven neurotransmitter-related candidate genes that were either associated with TS, OCD, or ASD (Abdulkadir et al. 2018).

\section{Genotyping and quality control}

Genotyping of 192 SNPs (Table S1) was performed on the Illumina GoldenGate Genotyping Assay for a subset of the cases $(N=464)$. Our sample was enriched by $N=122$ cases genotyped on the HumanOmniExpressExome v1.2 BeadChip genotyping array for a subset of the SNPs $\left(N_{\mathrm{SNPs}}=75\right)$ available on the Goldengate Assay and four SNPs that were not present on the Goldengate Assay. The total number of SNPs genotyped across both platforms
Table 1 Significant results from the main-effect analyses of previously implicated SNPs in relation to lifetime tic severity

\begin{tabular}{|c|c|c|c|c|c|c|c|c|}
\hline \multirow[t]{2}{*}{ SNP } & \multirow[t]{2}{*}{ Position } & \multirow[t]{2}{*}{ Chromosome } & \multirow[t]{2}{*}{ Gene } & \multirow[t]{2}{*}{ Category } & \multicolumn{2}{|c|}{$\begin{array}{l}\text { Main effect in } \\
\text { initial sample }\end{array}$} & \multicolumn{2}{|c|}{$\begin{array}{l}\text { Replication } \\
\text { main effect }\end{array}$} \\
\hline & & & & & $F$ & $P^{\mathrm{a}}$ & $F$ & $P^{\mathrm{a}}$ \\
\hline rs7123010 & $86,341,186$ & 11 & $M E 3$ & GWAS TS & 7.99 & $0.0004 *$ & 1.98 & 0.14 \\
\hline
\end{tabular}

SNP single-nucleotide polymorphism, GWAS genome-wide association study, TS Tourette syndrome *Significant after correcting for multiple testing $\left(P_{\text {all }}=0.0014\right)$

${ }^{a}$ Analyses were corrected for age, sex, and socioeconomic status 
was 196. Standard quality control checks were performed with PLINK (described in detail by Abdulkadir et al. 2018), which resulted in removal of 10 SNPs. We also removed SNPs with a genotype count less than $20(N=80$ SNPs $)$ and SNPs located on the $\mathrm{X}$ chromosome ( $N=8 \mathrm{SNPs})$ reducing the number of SNPs to 98 (Table S2).

\section{Statistical analyses}

We conducted case-only analyses of tic severity using linear regressions in $\mathrm{R}$ (corrected for age, sex, and SES) examining; (I) the main effects of the SNPs on tic severity; and (II) the interaction effect of these SNPs with a cumulative preand perinatal adversity score. SNPs were coded as $0=$ major allele homozygous (the reference category), $1=$ heterozygous, and $2=$ minor allele homozygous. Potential confounding due to relatedness of several cases was examined using mixed model analyses with familial relatedness as a random effect.

SNPs were selected from five a priori defined groups (Table S2) and we therefore applied correction for multiple testing, first, at the group level by dividing $P=0.05$ by the number of SNPs contained within each category; referred to as $P_{\text {group }}$ corrected. To correct for the number of groups tested, we further divided the obtained $P_{\text {group }}$ corrected by the number of groups (i.e., five) tested; referred to as the $P_{\text {all }}$. These groups were (I) top SNPs from GWAS of TS, $P_{\text {group }}$ corrected $=0.0071, P_{\text {all }}=0.0014$ (II) top SNPs from GWAS of OCD, ADHD, and ASD, $P_{\text {group }}$ corrected $=0.0063$, $P_{\text {all }}=0.0013$; (III) SNPs previously implicated in candidategene studies of TS $P_{\text {group }}$ corrected $=0.005, P_{\text {all }}=0.001$, (IV) SNPs previously implicated in OCD, or ASD, $P_{\text {group }}$ corrected $=0.0167, P_{\text {all }}=0.0033$; and $(\mathrm{V})$ tagging SNPs in neurotransmitter-related candidate genes, $P_{\text {group }}$ corrected $=0.0007, P_{\text {all }}=0.0001$. For SNPs that met the threshold of multiple testing, replication was sought in an independent sample (Scharf et al. 2013).

\section{Results}

\section{Sample description}

Cases missing clinical or demographic information $(N=68)$ were excluded, leaving 518 cases eligible for analyses. Results from the mixed model analyses in which a random intercept was included for familial relatedness gave similar results to the models without the random effect (Table S3, S4).

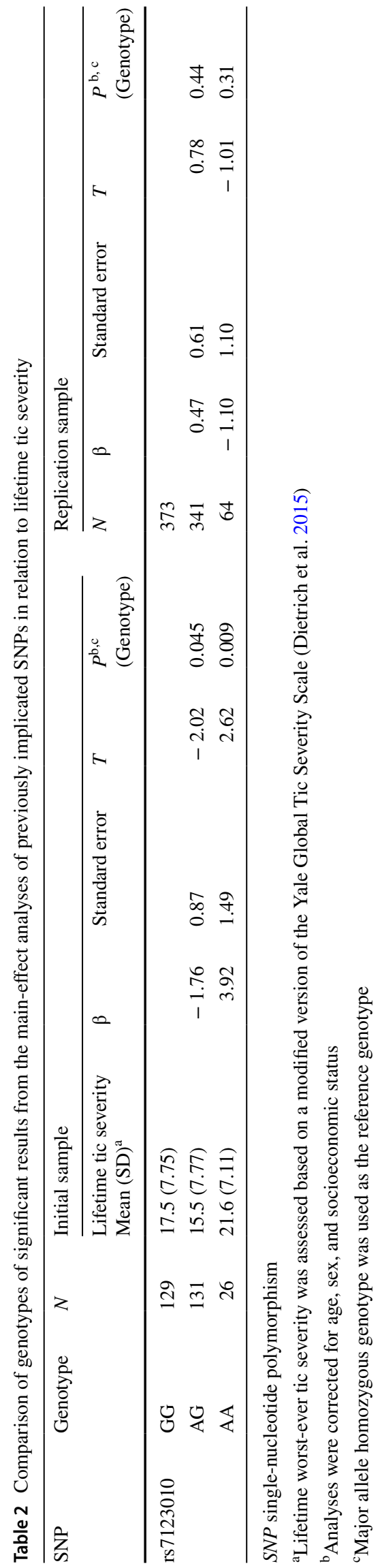




\section{Main effect SNPs}

We found a significant association between rs7123010, a top SNP from a GWAS of TS, and tic severity, also after correction for multiple testing ( $F=7.99, P=0.0004$; Tables 1,2 , and Table $\mathrm{S} 1$ ); the AA genotype was positively associated with tic severity. Results did not differ when we corrected for multiple comparisons using the less stringent Benjamini-Hochberg False Discover Rate.

\section{Gene-environment interaction}

We found a significant interaction of rs6539267, a top SNP from a TS GWAS $(F=6.80, P=0.001)$ with the cumulative pre- and perinatal adversity score, also after correction for multiple testing (Tables 3, 4; Fig. 1); the CC genotype along with a higher number of pre- and perinatal adversities was positively associated with tic severity (Table 4$)$. We found no significant interaction for rs7123010 ( $F=0.0197, P=0.98)$. For the GxE analysis, the pattern of results remained when we corrected for multiple comparisons using the less stringent Benjamini-Hochberg False Discover Rate.

\section{Replication rs7123010 and rs6539267}

Investigating the main effect of rs7123010 and the interaction between rs6539267 and the cumulative pre- and perinatal adversity score in the replication sample (Scharf et al. 2013) did not show a statistically significant association $(F=1.98, P=0.14)$ and $(F=1.29, P=0.28)$, respectively (Table 2).

\section{Discussion}

We investigated whether previously implicated SNPs (i) are associated with lifetime worst-ever tic severity and (ii) might interact with a cumulative pre- and perinatal adversity score previously reported to be associated with TS (Abdulkadir et al. 2016). We report a significant main effect of rs7123010 (a top TS GWAS SNP). We found no evidence for an interaction between rs7123010 and pre- and perinatal adversity. However, we did find a significant interaction between rs6539267 (another top TS GWAS SNP) and pre- and perinatal adversity. We could not confirm these findings in our replication sample (Scharf et al. 2013).

The SNP rs7123010 is located within the ME3 (Malic Enzyme 3) gene which encodes for a protein responsible for malate metabolism (Hsieh et al. 2019). The ME3 gene is reported to be expressed in several tissues including the brain, and the protein encoded by this gene is thought to be involved in several biological processes including fatty acid biosynthesis and insulin secretion (Hasan et al. 2015;

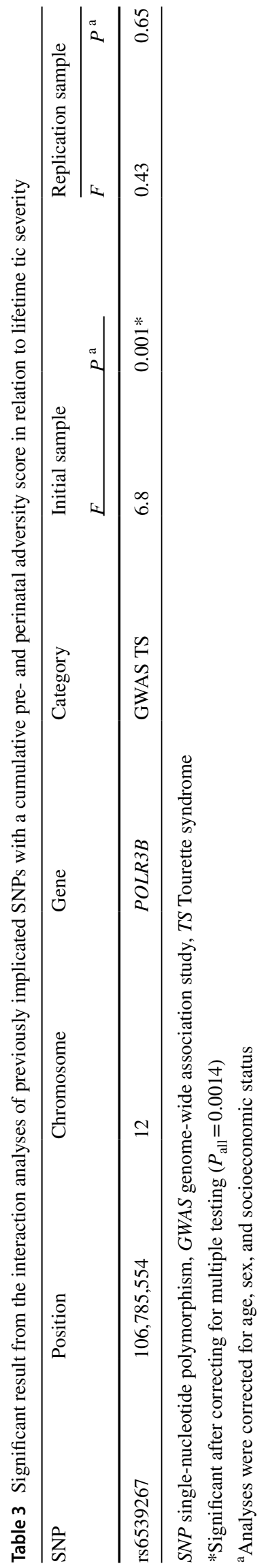


Hsieh et al. 2019). However, there is no evidence in the literature supporting a role of $M E 3$ in TS. The other significant SNP in this study, rs6539267, is located within the POLR3B gene that encodes for the second-largest catalytic subunit of RNA polymerase III, an enzyme involved in transcription of noncoding RNAs including transfer RNAs, small ribosomal RNAs, and microRNAs (Tétreault et al. 2011; Djordjevic et al. 2021). Mutations in POLR3B are reported to cause hypomyelinating leukodystrophy type 8 and the clinical presentations of these mutations are widespread and include ataxia, spasticity, variable intellectual disability and epilepsy, and demyelinating sensory motor peripheral neuropathy (Djordjevic et al. 2021). Despite the wide range of the clinical manifestations of POLR3B mutations, tics are not considered one of them.

A plausible explanation for the non-significant replication of rs7123010 and rs6539267 is that tic severity is likely a polygenic trait and that single SNPs only account for a small fraction of the total trait variance. Furthermore, statistical power could have also been an issue; the number of individuals with a homozygous genotype of the effect alleles was quiet low for SNPs rs7123010 (the AA genotype was present in about $10 \%$ of the individuals in the initial sample and the replication sample) and rs6539267 (the CC genotype was present in about $8 \%$ of the individuals in the initial sample and in $11 \%$ of the individuals in the replication sample).

This study benefitted from use of a well-characterized sample, and from the case-only design that has shown to have more power to detect gene-environment interactions than a case-control study (Kraft et al. 2007). Furthermore, using tic severity might have allowed the detection of small effects of SNPs that would have been otherwise missed when investigating caseness; e.g., a significant association for the Dopamine Transporter 13 ' variable number of tandem repeats has been found in relation to tic severity, but not in relation to the presence of TS (Tarnok et al. 2007).

Limitations of this study include the retrospective collection of lifetime tic severity and pre- and perinatal data, although evidence supports accurate maternal long-term recall of the latter (Rice et al. 2007). Measurement of lifetime tic severity differed across the study and replication samples, yet is not expected to explain current results. Finally, we cannot exclude that the investigated SNPs might interact with other environmental risk factors, such as life stress or infections.

In conclusion, the findings of this study suggest an association between rs7123010 and tic severity and potential gene-environment interactions of TS GWAS SNP rs6539267 with a cumulative pre- and perinatal adversity score in relation to tic severity. Our study highlights the future potential of common genetic risk variants in gene-environment studies in TS, perhaps through large-scale studies utilizing polygenic scores. 
Fig. 1 Interaction analyses of rs6539267 with a cumulative pre- and perinatal adversity score in relation to lifetime tic severity

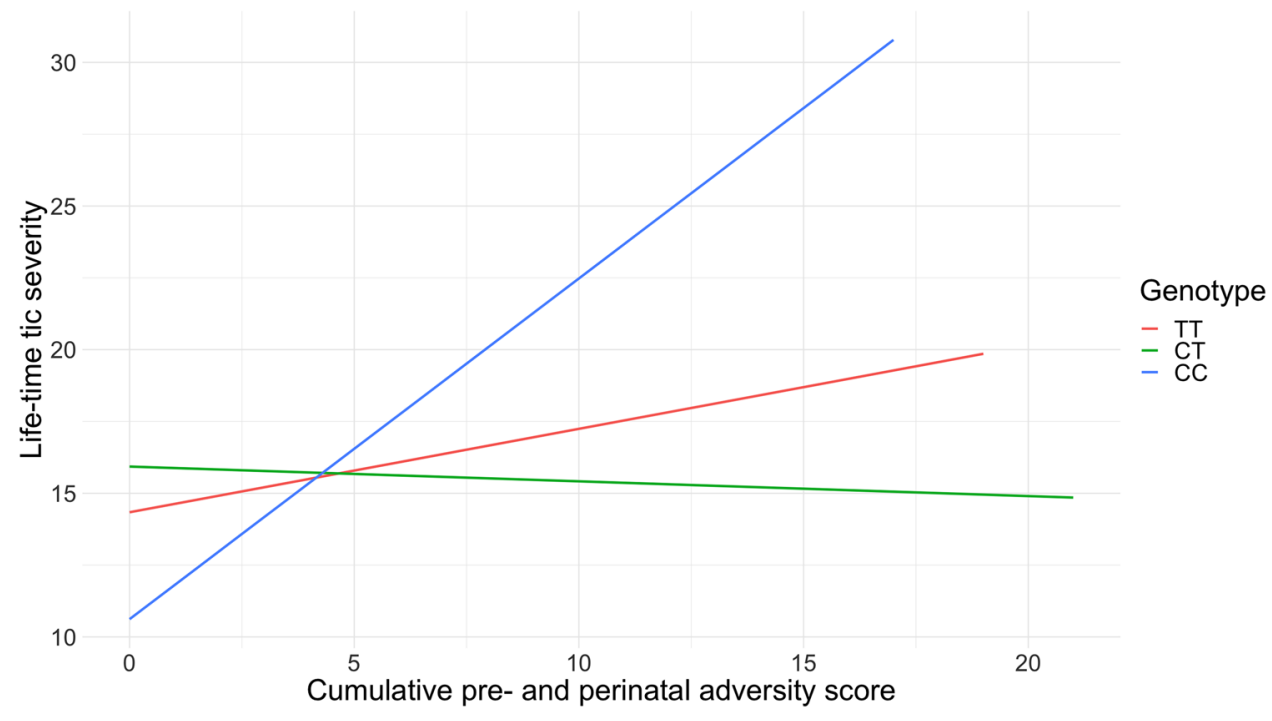

Supplementary Information The online version contains supplementary material available at https://doi.org/10.1007/s00702-021-02396-y.

Acknowledgements We wish to thank the families who have participated in and contributed to this study. We are grateful to New Jersey Center for Tourette Syndrome (NJCTS) for facilitating the inception and organization of the Tourette International Collaborative Genetics (TIC Genetics) study. We would like to thank the members of the TIC Genetics study which are: Yana Bromberg, Lawrence W. Brown, Keun-Ah Cheon, Barbara J. Coffey, Li Deng, Shan Dong, Thomas V. Fernandez, Blanca Garcia-Delgar, Erika Gedvilaite, Donald L. Gilbert, Dorothy E. Grice, Julie Hagstrøm, Tammy Hedderly, Isobel Heyman,Hyun Ju Hong, Chaim Huyser, Laura Ibanez-Gomez, Young Key Kim, Young Shin Kim, Robert A. King, Yun-Joo Koh, Sodahm Kook, Samuel Kuperman, Bennett Leventhal, Marcos Madruga-Garrido, Jeffrey D. Mandell, Athanasios Maras, Pablo Mir, Astrid Morer, Alexander Münchau, Cara Nasello, Kerstin J. Plessen, Petra Richer, Veit Roessner, Stephan Sanders, Eun-Young Shin, Louw Smith, DongHo Song, Jungeun Song, Matthew W. State, Nawei Sun, Frank Visscher, Michael F. Walker, Shuoguo Wang, Jeremy Willsey, Jinchuan Xing, Yeting Zhang, Anbo Zhou, and Samuel H. Zinner. We would also wish to thank the Tourette Association of America International Consortium for Genetics (TAAICG) for providing data that was used as replication of the findings of this study. Members of the TAAICG are: Cathy L. Barr, James R. Batterson, Cheston Berlin, Ruth D. Bruun, Cathy L. Budman, Danielle C. Cath, Sylvain Chouinard, Giovanni Coppola, Nancy J. Cox, Sabrina Darrow, Lea K. Davis, Yves Dion, Nelson B. Freimer, Marco A. Grados, Matthew E. Hirschtritt, Alden Y. Huang, Cornelia Illmann, Robert A. King, Roger Kurlan, James F. Leckman, Gholson J. Lyon, Irene A. Malaty, Carol A. Mathews, William M. MaMahon, Benjamin M. Neale, Michael S. Okun, Lisa Osiecki, David L. Pauls, Danielle Posthuma, Vasily Ramensky, Mary M. Robertson, Guy A. Rouleau, Paul Sandor, Jeremiah M. Scharf, Harvey S. Singer, Jan Smit, Jae-Hoon Sul, and Dongmei Yu.

Author contributions MA, GAH, $\mathrm{PJH}$, and $\mathrm{AD}$ were involved in the organization, design, and execution, critique, and the statistical analysis of the research project. CAM, JMS, DY, and LO were involved in the replication effort of the findings in this study. MA wrote the first draft of the manuscript, which was critically reviewed by JAT, GAH, $\mathrm{PJH}$, and $\mathrm{AD}$ who were also involved in the conception of the research project. All authors were involved in the review and critique of the manuscript. All authors have approved the final article.
Funding This research was funded by National Institute of Mental Health (NIMH) grant R01MH092293 (to GAH and JAT) and NJCTS (New Jersey Center for Tourette Syndrome and Associated Disorders; to GAH and JAT). This work was also supported by grants from the Judah Foundation, the Tourette Association of America, National Institute of Health (NIH) Grants NS40024, NS016648, MH079489, MH073250, the American Recovery and Re-investment Act (ARRA) Grants NS040024-07S1; NS16648-29S1; NS040024-09S1; MH092289; MH092290; MH092291; MH092292; R01MH092293; MH092513; MH092516; MH092520; MH071507; MH079489; MH079487; MH079488; and MH079494. Dr. Mir has received grants from the Instituto de Salud Carlos III (PI10/01674, PI13/01461), the Consejería de Economía, Innovación, Ciencia y Empresa de la Junta de Andalucía (CVI-02526, CTS-7685), the Consejería de Salud y Bienestar Social de la Junta de Andalucía (PI-0741/2010, PI-0437-2012, PI-0471-2013), the Sociedad Andaluza de Neurología, the Fundación Alicia Koplowitz, the Fundación Mutua Madrileña and the Jaques and Gloria Gossweiler Foundation. Dr. Morer has received grants from the Fundacion Alicia Koplowitz and belongs to the research group of the Comissionat per Universitats i Recerca del Departmanent d'Innovacio (DIUE) 2009SGR1119. Dr. Münchau has received grants from the Deutsche Forschungsgemeinschaft (DFG: MU 1692/3-1, MU 1692/4-1 and FOR 2698). This study was also supported by a Grant from the National Institute for Environmental Health Science (R01 ES021462).

Availability of data and materials The clinical data and biomaterials (DNA, transformed cell lines, RNA) are part of a sharing repository located within the National Institute for Mental Health Center for Collaborative Genomics Research on Mental Disorders, USA, and are available to the broad scientific community: https://www.ncbi.nlm. nih.gov/projects/gap/cgi-bin/study.cgi?study_id=phs001423.v2.p2.

Code availability Not applicable.

\section{Declarations}

Conflict of interest Drs. Mathews and Scharf are on the scientific advisory board of the Tourette Association of America (TAA) and have received travel and Grant support from the TAA. Dr. Mathews is also on the scientific advisory board of the International Obsessive-Compulsive Disorder Foundation and the Family Foundation for OCD Research. Dr. Scharf is on the scientific advisory board of the TLC 
Foundation for Body-Focused Repetitive Behaviors and has received consulting fees from Nuvelution Pharma and Abide Pharmaceuticals. Dr. Coffey is co-Chair of the TAA Medical Advisory Board and has received honoraria from the TAA-CDC partnership She has also received honoraria from the American Academy of Child and Adolescent Psychiatry, Partners Health Care, Harvard Medical School/Psychiatry Academy; consulting fees fromTeva/Nuvelution, and Skyland Trail, and research support from NIMH and Emalex Pharmaceuticals. The remaining authors reported no biomedical financial interest or potential conflict of interest.

Ethics approval All adult participants and parents of children provided written informed consent along with written or oral assent of their participating child. The Institutional Review Board of each participating site had approved the study which are: 1. University of Groningen 2. Yale Child Study Center 3. Childrens Hospital of Philadelphia 4. Yonsei University College of Medicine 5. Icahn School of Medicine at Mount Sinai 6. Nathan S. Kline Institute for Psychiatric Research 7. Yulius Mental Health Organization 8. Medizinische Hochschule Hannover Klinik fur Psychiatrie 9. University Hospital Medical Center Hamburg-Eppendorf 10. Hospital Clinic Universitari 11. Cincinnati Childrens Hospital Medical Center 12. Evelina London Childrens Hospital GSTT 13. Great Ormond Street Hospital for Children and UCL Institute of Child Health 14. Hallym University Sacred Heart Hospital 15. De Bascule 16. Amsterdam Academic Medical Center 17. Yonsei Bom Clinic 18. University of California San Francisco 19. Korea Institute for Childrens Social Development 20. Kangbuk Samsung Hospital 21. University of Iowa Carver College of Medicine Iowa City 22. University of Ulm 23. Universidad de Sevilla 24. Erasmus Medical Center-Sophia Childrens Hospital Rotterdam 25. Institut de Investigacions Biomediques August Pi i Sunyer (IDIPABS) and Centro de Investigacion en Red de Salud Mental (CIBERSAM) 26. University of Lubeck 27. University of Copenhagen 28. TU Dresden 29. National Health Insurance Service Ilsan Hospital 30. Altrecht Institute for Mental Health 31. Admiraal De Ruyter Ziekenhuis 32. University of Washington Seattle.

\section{Consent to participate As stated above.}

Consent for publication All participants agreed on the use and publication of anonymized data.

Open Access This article is licensed under a Creative Commons Attribution 4.0 International License, which permits use, sharing, adaptation, distribution and reproduction in any medium or format, as long as you give appropriate credit to the original author(s) and the source, provide a link to the Creative Commons licence, and indicate if changes were made. The images or other third party material in this article are included in the article's Creative Commons licence, unless indicated otherwise in a credit line to the material. If material is not included in the article's Creative Commons licence and your intended use is not permitted by statutory regulation or exceeds the permitted use, you will need to obtain permission directly from the copyright holder. To view a copy of this licence, visit http://creativecommons.org/licenses/by/4.0/.

\section{References}

Abdulkadir M, Tischfield JA, King RA et al (2016) Pre- and perinatal complications in relation to Tourette syndrome and co-occurring obsessive-compulsive disorder and attention-deficit/hyperactivity disorder. J Psychiatr Res 82:126-135. https://doi.org/10.1016/j. jpsychires.2016.07.017
Abdulkadir M, Londono D, Gordon D et al (2018) Investigation of previously implicated genetic variants in chronic tic disorders: a transmission disequilibrium test approach. Eur Arch Psychiatry Clin Neurosci 268:301-316. https://doi.org/10.1007/ s00406-017-0808-8

Anney R, Klei L, Pinto D et al (2012) Individual common variants exert weak effects on the risk for autism spectrum disorderspi. Hum Mol Genet 21:4781-4792. https://doi.org/10.1093/hmg/ dds301

Brander G, Rydell M, Kuja-Halkola R et al (2018) Perinatal risk factors in Tourette's and chronic tic disorders: a total population sibling comparison study. Mol Psychiatry 23:1189-1197. https://doi.org/ $10.1038 / \mathrm{mp} .2017 .31$

Comings DE, Comings BG, Muhleman D et al (1991) The dopamine D2 receptor locus as a modifying gene in neuropsychiatric disorders. J Am Med Assoc 266:1793-1800. https://doi.org/10.1001/ jama.266.13.1793

Dietrich A, Fernandez TV, King R et al (2015) The Tourette international collaborative genetics (TIC Genetics) study, finding the genes causing Tourette syndrome: objectives and methods. Eur Child Adolesc Psychiatry. https://doi.org/10.1007/ s00787-014-0543-x

Djordjevic D, Pinard M, Gauthier M-S et al (2021) De novo variants in POLR3B cause ataxia, spasticity, and demyelinating neuropathy. Am J Hum Genet 108:186-193. https://doi.org/10.1016/j.ajhg. 2020.12 .002

Hasan NM, Longacre MJ, Stoker SW et al (2015) Mitochondrial malic enzyme 3 is important for insulin secretion in pancreatic $\beta$-cells. Mol Endocrinol 29:396-410. https://doi.org/10.1210/me. 2014-1249

Hinney A, Scherag A, Jarick I et al (2011) Genome-wide association study in German patients with attention deficit/hyperactivity disorder. Am J Med Genet B Neuropsychiatr Genet 156B:888-897. https://doi.org/10.1002/ajmg.b.31246

Hsieh J-Y, Shih W-T, Kuo Y-H et al (2019) Functional roles of metabolic intermediates in regulating the human mitochondrial NAD(P)+-dependent malic enzyme. Sci Rep 9:9081. https://doi. org/10.1038/s41598-019-45282-0

Kraft P, Yen YC, Stram DO et al (2007) Exploiting gene-environment interaction to detect genetic associations. Hum Hered 63:111-119. https://doi.org/10.1159/000099183

Mathews C, Scharf JM, Miller LL et al (2014) Association between pre- and perinatal exposures and Tourette syndrome or chronic tic disorder in the ALSPAC cohort. Br J Psychiatry 204:40-45. https://doi.org/10.1192/bjp.bp.112.125468

Mick E, McGough J, Loo S et al (2011) Genome-wide association study of the child behavior checklist dysregulation profile. J Am Acad Child Adolesc Psychiatry. https://doi.org/10.1016/j.jaac. 2011.05.001

Paschou P, Yu D, Gerber G et al (2014) Genetic association signal near NTN4 in Tourette syndrome. Ann Neurol 76:310-315. https://doi. org/10.1002/ana.24215

Qi Y, Zheng Y, Li Z, Xiong L (2017) Progress in genetic studies of Tourette's syndrome. Brain Sci. https://doi.org/10.3390/brain sci7100134

Rice F, Lewis A, Harold G et al (2007) Agreement between maternal report and antenatal records for a range of pre and peri-natal factors: the influence of maternal and child characteristics. Early Hum Dev 83:497-504. https://doi.org/10.1016/j.earlhumdev.2006. 09.015

Robertson MM, Eapen V, Singer HS et al (2017) Gilles de la Tourette syndrome. Nat Rev Dis Prim 3:16097. https://doi.org/10.1038/ nrdp. 2016.97 
Scharf JM, Yu D, Mathews C et al (2013) Genome-wide association study of Tourette's syndrome. Mol Psychiatry 18:721-728. https:// doi.org/10.1038/mp.2012.69

Stewart SE, Yu D, Scharf JM et al (2013) Genome-wide association study of obsessive-compulsive disorder. Mol Psychiatry 18:788798. https://doi.org/10.1038/mp.2012.85

Tarnok Z, Ronai Z, Gervai J et al (2007) Dopaminergic candidate genes in Tourette syndrome: association between tic severity and 3' UTR polymorphism of the dopamine transporter gene. Am J Med Genet B Neuropsychiatr Genet 144B:900-905. https://doi. org/10.1002/ajmg.b.30517

Tétreault M, Choquet K, Orcesi S et al (2011) Recessive mutations in POLR3B, encoding the second largest subunit of pol iii, cause a rare hypomyelinating leukodystrophy. Am J Hum Genet 89:652655. https://doi.org/10.1016/j.ajhg.2011.10.006
Walkup J, Leckman J (1988) Modified schedule for risk and protective factors early in development. Child Study Center, Yale University, New Haven

Wang K, Zhang H, Ma D et al (2009) Common genetic variants on 5p14.1 associate with autism spectrum disorders. Nature 459:528533. https://doi.org/10.1038/nature07999

Yu D, Sul JH, Tsetsos F et al (2019) Interrogating the genetic determinants of tourette's syndrome and other tic disorders through genome-wide association studies. Am J Psychiatry 176:217-227. https://doi.org/10.1176/appi.ajp.2018.18070857

Publisher's Note Springer Nature remains neutral with regard to jurisdictional claims in published maps and institutional affiliations.

\section{Authors and Affiliations}

Mohamed Abdulkadir ${ }^{1,2}$ (D) Dongmei Yu ${ }^{3,4} \cdot$ Lisa Osiecki $^{4} \cdot$ Robert A. King $^{5} \cdot$ Thomas V. Fernandez $^{5}$. Lawrence W. Brown $^{6} \cdot$ Keun-Ah Cheon $^{7}$ - Barbara J. Coffey ${ }^{8,9,10}$. Blanca Garcia-Delgar ${ }^{11}$. Donald L. Gilbert ${ }^{12}$. Dorothy E. Grice ${ }^{8}$. Julie Hagstrøm ${ }^{13} \cdot$ Tammy Hedderly $^{14}$. Isobel Heyman ${ }^{15}$. Hyun Ju Hong ${ }^{16}$. Chaim Huyser ${ }^{17}$. Laura Ibanez-Gomez ${ }^{18}$. Young Key Kim ${ }^{19}$. Young-Shin Kim ${ }^{20} \cdot$ Yun-Joo Koh ${ }^{21}$. Sodahm Kook ${ }^{22}$. Samuel Kuperman ${ }^{23} \cdot$ Bennett Leventhal ${ }^{20} \cdot$ Marcos Madruga-Garrido $^{24} \cdot$ Athanasios Maras $^{25} \cdot$ Pablo Mir $^{26}$. Astrid Morer ${ }^{27}$. Alexander Münchau ${ }^{28} \cdot$ Kerstin J. Plessen ${ }^{13} \cdot$ Veit Roessner $^{29}$. Eun-Young Shin ${ }^{7}$. Dong-Ho Song ${ }^{30}$. Jungeun Song ${ }^{31}$. Frank Visscher ${ }^{32}$. Samuel H. Zinner ${ }^{33}$. Carol A. Mathews ${ }^{34}$. Jeremiah M. Scharf ${ }^{3,4}$. Jay A. Tischfield ${ }^{2}$. Gary A. Heiman ${ }^{2} \cdot$ Andrea Dietrich $^{1} \cdot$ Pieter J. Hoekstra ${ }^{1}$

1 Department of Child and Adolescent Psychiatry, University Medical Center Groningen, Hanzeplein 1, 9713 GZ Groningen, The Netherlands

2 Department of Genetics and the Human Genetics Institute of New Jersey, Rutgers, The State University of New Jersey, Piscataway, NJ 08854, USA

3 Department of Neurology, Center for Genomic Medicine, Massachusetts General Hospital, Harvard Medical School, Boston, MA 02114, USA

4 Department of Psychiatry, Psychiatric and Neurodevelopmental Genetics Unit, Massachusetts General Hospital, Harvard Medical School, Boston, MA 02114, USA

5 Yale Child Study Center, Yale University School of Medicine, New Haven, CT 06510, USA

6 Pediatric Neuropsychiatry Program, Division of Neurology, The Children's Hospital of Philadelphia, Philadelphia, PA 19104, USA

7 Yonsei University College of Medicine, Severance Hospital, Seoul 120-752, South Korea

8 Icahn School of Medicine at Mount Sinai, New York, NY, USA

9 Nathan S. Kline Institute for Psychiatric Research, Orangeburg, NY, USA

10 University of Miami Miller School of Medicine, Miami, FL 33146, USA

11 Department of Child and Adolescent Psychiatry and Psychology, Institute of Neurosciences, Hospital Clinic Universitari, Barcelona, Spain
12 Cincinnati Children's Hospital Medical Center, Cincinnati, $\mathrm{OH}, \mathrm{USA}$

13 Child and Adolescent Mental Health Center, Mental Health Services, Capital Region of Denmark and Faculty of Health Sciences, University of Copenhagen, Copenhagen, Denmark

14 Evelina London Children's Hospital GSTT, Kings Health Partners AHSC, London, UK

15 Psychological Medicine, UCL Great Ormond Street Institute of Child Health, London, UK

16 Department of Psychiatry, Hallym University Sacred Heart Hospital, Anyang, Gyeonggi 14068, South Korea

17 Academic Center for Child and Adolescent Psychiatry De Bascule, 1105 AZ Amsterdam, The Netherlands

18 Family Health Centers at NYU Langone, Brooklyn, NY 11220, USA

19 Department of Psychiatry, Yonsei Bom Clinic, Seoul 03330, South Korea

20 University of California San Francisco Medical Center, San Francisco, CA 94143, USA

21 Korea Institute for Children's Social Development, Seoul, South Korea

22 Yonsei-Nuri Mental Health Clinic, Seoul 08005, South Korea

23 Department of Psychiatry, Carver College of Medicine, University of Iowa, Iowa, IA 52242, USA

24 Sección de Neuropediatría, Instituto de Biomedicina de Sevilla, Hospital Universitario Virgen del Rocío/CSIC/Universidad de Sevilla, Seville, Spain 
25 Yulius Academy, Yulius Mental Health Organization, 3311 JG Dordrecht, The Netherlands

26 Unidad de Trastornos del MovimientoInstituto de Biomedicina de Sevilla (IBiS). Hospital Universitario Virgen del Rocío/CSICUniversidad de Sevilla, Seville, Spain

27 Department of Child and Adolescent Psychiatry and Psychology, Institute of Neurosciences, Hospital Clinic Universitari Barcelona, Spain; Institut d'Investigacions Biomediques August Pi i Sunyer (IDIPABS) and Centro de Investigacion en Red de Salud Mental (CIBERSAM), Barcelona, Spain 23562 Lübeck, Germany

29 Department of Child and Adolescent Psychiatry, TU Dresden, Dresden, Germany
30 Yonsei University Severance Hospital, Seoul 03722, South Korea

31 Department of Psychiatry, National Health Insurance Service Ilsan Hospital, Goyang, Gyeonggi 10444, South Korea

32 Admiraal De Ruyter Ziekenhuis, Department of Neurology, Goes, The Netherlands

33 Department of Pediatrics, Division of Developmental Medicine, University of Washington School of Medicine, 1925 NE Pacific Street, Box 356524, Seattle, WA 98195, USA

34 Department of Psychiatry, Center for OCD, Anxiety and Related Disorders, and Genetics Institute, University of Florida College of Medicine, Gainesville, FL 32611, USA 